\title{
Molecular markers for quantification of bioactive fungal strains associated with Pinus radiata
}

\author{
K. Mellow ${ }^{1}$, P. Chettri ${ }^{1}$, S. Kabir ${ }^{1}$, R.L. McDougal ${ }^{2}$, M.P. Cox ${ }^{1}$, A. Stewart ${ }^{2}$, \\ C. Lange ${ }^{3}$, J.M. Steyaert ${ }^{3}$ and R.E. Bradshaw ${ }^{1}$ \\ ${ }^{1}$ Institute of Fundamental Sciences, Massey University, Palmerston North 4442, New Zealand \\ ${ }^{2}$ Scion, 49 Sala Street, Rotorua 3046, New Zealand \\ ${ }^{3}$ Bio-Protection Research Centre, PO Box 85084, Lincoln University, Lincoln 7647, New Zealand \\ Corresponding author: r.e.bradshaw@massey.ac.nz
}

\begin{abstract}
Trichoderma cf. atroviride is an endophytic soil fungus, which has been the target of much research due to its plant growth promoting effects and use as a biocontrol agent. For specific bioactive strains of this species to be used with long-lived forest trees such as Pinus radiata, they must persist over the long term in the host root system. To investigate the persistence of specific $T$. $c f$. atroviride strains in the roots of $P$. radiata, unique regions were identified in the genomes of strains that were more than $99.7 \%$ identical by using next generation sequencing. Based on these unique regions, probe-based, strain-specific quantitative-PCR assays were developed. The assays can be used to test strain persistence in nursery and forest-grown seedlings inoculated with mixtures of $T$. $c f$. atroviride strains, as well as in laboratory experiments to determine the effect of these strains on plant metabolism and defence.
\end{abstract}

Keywords Trichoderma, Pinus radiata, quantitative PCR, next-generation sequencing, bioinoculants

\section{INTRODUCTION}

Trichoderma spp. are common soil fungi that have become targets of research in bioprotection because strains of some species exhibit biocontrol activity, with some marketed as biopesticides (Harman 2011). Protection against pathogens in hosts inoculated with Trichoderma can result from direct antagonism and antibiosis within the rhizosphere, or indirect protection via the enhancement of host resistance to pathogen attack (Harman 2011). Certain species of Trichoderma have also been shown to promote plant growth.
For example, both growth and health benefits were shown for Pinus radiata seedlings treated with T. hamatum (Hohmann et al. 2011).

A decisive factor before deploying specific strains of Trichoderma spp. in forests is whether they are able to persist and become established in the soil and roots, potentially providing long-term protection and possibility other benefits. Because Trichoderma spp. are ubiquitous and commonly found in soil, to answer this question it is first necessary to develop 
molecular tools that can identify and quantify specific strains shown to have a bio-protective effect amongst the Trichoderma population.

The aim of this study was to develop sensitive and specific probe-based, real-time PCR assays for two strains of Trichoderma cf. atroviride (R33 and R84) that were shown to promote growth in $P$. radiata (Reglinski et al. 2012) and to trial the use of these assays with $P$. radiata root samples. To achieve specificity, primers tested in this assay were designed to target unique single nucleotide polymorphisms that were detected using genome sequencing of $T$. cf. atroviride strains.

\section{MATERIALS AND METHODS \\ Sequencing and identification of single nucleotide polymorphisms (SNPs)}

T. cf. atroviride strains R33 and R84, along with two other strains of the same species, R32 and R40 (Lincoln University, New Zealand; Lange 2015) were grown in potato dextrose broth (Difco) for 3 days at $25^{\circ} \mathrm{C}$ and genomic DNA extracted using a Gentra ${ }^{\circledR}$ Puregene ${ }^{\mathrm{Tm}}$ Tissue kit (Qiagen, Netherlands). Genomic DNA was sequenced using an Illumina GAII platform (Massey University Genome Service, New Zealand). The reads (>11 million per strain) were mapped to the reference genome of $T$. atroviride IMI206040 (Joint Genome Institute; http:// genome.jgi-psf.org/Triat2/Triat2.home.html) using the Burrows-Wheeler transform (BWT) algorithm and the program bwa v.0.5.8 (Li \& Durbin 2009). The strains were sequenced with a mean of 15-35 reads per nucleotide position and $73-87 \%$ representation of the reference genome. Amongst the mapped reads, SNPs unique to R33 or R84 were identified using SAMtools (Li et al. 2009) and custom code, using a threshold of at least eight reads at those sites in every sequenced strain.

\section{PCR conditions}

Primers and fluorescent probes for real-time PCR were designed to the selected SNP regions using AlleleID ${ }^{\circ}$ software (http://www.premierbiosoft. com) (Table 1). The 5' and 3' ends of R33- and R84-specific probes were labelled with 6-carboxyfluorescein (FAM) and quencher dye BHQ-1 respectively. For a normalisation control, the 5 ' and 3' ends of the Pinus radiata cinnamyl alcohol dehydrogenase $(\mathrm{CAD})$ gene probe were labelled with HEX (5') and BHQ-1 (3'), respectively (Chettri et al. 2012). Real-time PCR was carried out using the quantitative PCR (qPCR) protocol of Chettri et al. (2012). The reaction was performed with a LightCycler ${ }^{\circledast} 480$ Probes system (Roche Applied Science, Penzberg, Germany) where each $10 \mu$ of reaction contained: $5 \mu$ l of $2 \times$ Probe master mix, $1 \mu \mathrm{l} 10 \times$ primer probe mix (containing $2 \mu \mathrm{M}$ of the appropriate probe and $4 \mu \mathrm{M}$ of each specific primer), $2 \mu \mathrm{l}$ water and $2 \mu \mathrm{DNA}$ template. PCR

Table 1 Primer and probe sequences.

\begin{tabular}{|c|c|c|}
\hline Target & Primer/probe & Sequence (5' to $\left.3^{\prime}\right)$ \\
\hline P. radiata & CAD918 & CAGCAAGAGGATTTGGACCTA \\
\hline \multirow[t]{2}{*}{$C A D$ gene $^{1}$} & CAD1019 & TTCAATACCCACATCTGATCAAC \\
\hline & Probe945 & HEX-TGTGAACCATGACGGCACCC-BHQ1 \\
\hline \multirow{3}{*}{$\begin{array}{l}\text { T. cf. } \\
\text { atroviride } \\
\text { R33 }\end{array}$} & RM5Ka & GCAAGTTGGATACAGTTG \\
\hline & RM6Ka & CCAGTAACTAGAATCGCA \\
\hline & ProbeTrichKa & 6FAM-TTGTATTAGTCCCACTCTATCAAG-BHQ1 \\
\hline \multirow{3}{*}{$\begin{array}{l}\text { T. cf. } \\
\text { atroviride } \\
\text { R84 }\end{array}$} & RM1 & CACTTCCAACTTATTTTCTGCC \\
\hline & RM2 & CCGTACATGATGCCTCACA \\
\hline & ProbeLu584Pc & 6FAM-CCACCAACTAATAGACGCACCG- BHQ1 \\
\hline
\end{tabular}

${ }^{1}$ Chettri et al. 2012 
was conducted at $95^{\circ} \mathrm{C}$ for $10 \mathrm{~min}$ followed by 55 cycles of: denaturation at $95^{\circ} \mathrm{C}$ for $10 \mathrm{~s}$, annealing at $58^{\circ} \mathrm{C}$ for $15 \mathrm{~s}$, extension at $72^{\circ} \mathrm{C}$ for $20 \mathrm{~s}$, followed by $40^{\circ} \mathrm{C}$ for $10 \mathrm{~s}$. All real-time PCR runs included negative and positive controls, with no template or purified target template, respectively.

\section{Assessment of specificity, sensitivity and efficiency}

To assess primer specificity, genomic DNA was extracted from a range of T. cf. atroviride and T. atroviride strains, including R32 and R40 (Lange 2015) as well as from other common forest and soil fungi including Cyclaneusma minus, Lophodermium pinastri, Nectria fuckeliana, Sclerotinia sclerotiorum and Rhizoctonia solani. The quality of template DNA was checked by PCR using universal ribosomal spacer (ITS) primers as described previously (Bradshaw et al. 2000), then used in duplicate real-time PCR reactions with the R33- and R84-specific primer/probe combinations.

To assess the sensitivity of the probe-based PCR method, and to provide standard curves for quantification, qPCR reactions were carried out with a 5-fold dilution series of template DNA ranging from $0.1 \mathrm{pg}$ to $10 \mathrm{ng}$ for each of R33 and R84. For normalisation with pine DNA, a standard curve was similarly prepared using from $32 \mathrm{pg}$ to 100 ng $P$. radiata DNA extracted from freeze-dried root samples using a Genomic DNA Mini Plant Kit (Geneaid Biotech Ltd., New Taipei City, Taiwan). Reactions were run in triplicate. Root tissue was obtained from clones (cuttings) of $P$. radiata that had been treated by flooding the soil at the potting stage with either a mixed spore suspension of $T$. cf. atroviride $\mathrm{R} 33$ and $\mathrm{R} 84$ (treatment), or with water (control). Abundance of the specific T. $c f$. atroviride DNA in pine roots, as determined by qPCR ( 5 biological and 2 technical replicates), was normalised by the amount of $P$. radiata DNA by using both fungal (R33 or R84) and plant (CAD) primers and probes. Statistical analyses involved regression analysis of the relationship between log DNA concentration and quantification cycle $\left(\mathrm{C}_{\mathrm{q}}\right)$ in the standard curves, and a two-tailed Student's t-test to compare levels of T. cf. atroviride

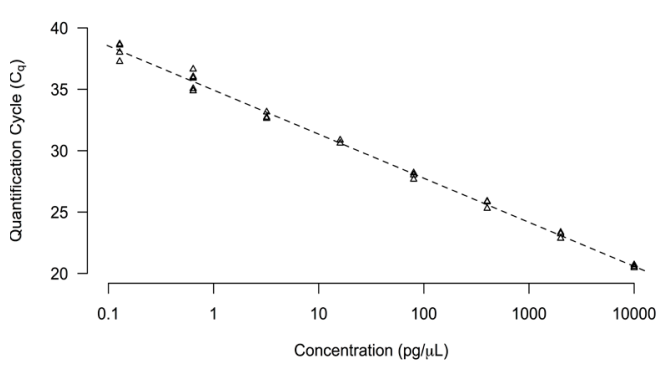

Figure 1 A standard curve for quantifying T. $c f$. atroviride strain R33, showing the concentration of serially diluted R33 DNA against the PCR quantification cycle $\left(\mathrm{C}_{\mathrm{q}}\right)$ required to amplify the fluorescent signal above a fixed threshold. The lowest limit of detection within the linear range was $\sim 0.1$ pg per reaction.

in treated and control $P$. radiata roots.

\section{RESULTS AND DISCUSSION Primer development and specificity}

Genome sequence data suggested that R33 and R84 were $>99.7 \%$ identical to each other and to the other two T. cf. atroviride strains sequenced, with estimated genome sizes of $36 \mathrm{Mb}$. From among the variable regions, more than 16,000 putative SNPs were identified for each of R33 and R84. From these, two regions were selected for the development of diagnostic markers. Primers and probes were designed to one region specific for each of R33 and R84 (Table 1). Cross-template PCR reactions (i.e., R84 template with R33 primers/probe and vice-versa) did not show any amplification, indicating strain-specificity of the primers and probes. Specificity of the R33 or R84 primer/probe sets was further shown by a lack of PCR amplification with template DNA from either P. radiata or other fungal samples, including R32 and R40.

\section{Assay sensitivity and PCR amplification efficiency}

Standard curves prepared by amplifying a range of R33 and R84 template DNA concentrations 
showed amplification efficiencies of 1.93 for both strains, close to the expected value of 2.0 (the amount of target DNA is doubled in each round of PCR). An example standard curve is shown in Figure 1. Regression $\left(\mathrm{R}^{2}\right)$ values of $0.995,0.986$ and 0.988 for R33, R84 and pine CAD targets, respectively, showed a linear correlation between quantification cycle $\left(\mathrm{C}_{\mathrm{q}}\right)$ and $\log$ concentration over five orders of magnitude in each case. The lower limit of detection of R33 and R84 within the linear range of the standard curves was $0.1 \mathrm{pg}$ of target DNA per reaction; based on a genome size of $36 \mathrm{Mb}$, this corresponds to just under 3 nuclei.

P. radiata root samples treated with $T$. $c f$. atroviride were shown to have $0.79 \pm 0.36 \mathrm{pg} / \mu \mathrm{l}$ R33 and $0.73 \pm 0.12 \mathrm{pg} / \mu \mathrm{l} 84$, and normalised ratios to pine DNA of $1.7 \times 10^{-5}$ and $1.5 \times 10^{-5}$, respectively, significantly higher than untreated controls with $0.00 \mathrm{pg} / \mu \mathrm{l}(\mathrm{P}<0.01)$ and well above the lower limit of detection.

\section{CONCLUSIONS}

Using an approach based on whole-genome sequencing to identify polymorphisms between two strains of T. cf. atroviride, a strainspecific, probe-based quantitative real-time PCR assay was developed. This assay will underpin the development of a testing system to check the durability of Trichoderma bioinoculants in the forest environment, and will be used in laboratory studies that aim to determine how pine defence is induced by these strains.

\section{ACKNOWLEDGEMENTS}

Robert Hill (Lincoln University) and Rebecca Ganley (Scion) are thanked for providing samples. Ruby Roach (Massey University) is thanked for laboratory assistance. This work was funded by AGMARDT and the Forest Owners Association of New Zealand.

\section{REFERENCES}

Bradshaw RE, Ganley RJ, Jones WT, Dyer PS 2000. High levels of dothistromin toxin produced by the forest pathogen Dothistroma pini. Mycological Research 104: 325-332.

Chettri P, Calvo AM, Cary JW, Dhingra S, Guo Y, McDougal RL, Bradshaw RE 2012. The veA gene of the pine needle pathogen Dothistroma septosporum regulates sporulation and secondary metabolism. Fungal Genetics and Biology 49: 141-151.

Harman GE 2011. Multifunctional fungal plant symbionts: new tools to enhance plant growth and productivity. New Phytologist 189: 647-649.

Hohmann P, Jones EE, Hill RA, Stewart A 2011. Understanding Trichoderma in the root system of Pinus radiata: associations between rhizosphere colonisation and growth promotion for commercially grown seedlings. Fungal Biology, 115: 759-767.

Lange C 2015. The genome and beyond: Phenotypic determinants of two Trichoderma cf. atroviride sister strains. $\mathrm{PhD}$ Thesis, Lincoln University.

Li H, Durbin R 2009. Fast and accurate short read alignment with Burrows-Wheeler Transform. Bioinformatics 25: 1754-1760.

Li H, Handsaker B, Wysoker A, Fennell T, Ruan J, Homer N, Marth G, Abecasis G, Durbin R and 1000 Genomes Project Data Processing Subgroup 2009. The sequence alignment/ map format and SAMtools. Bioinformatics 25: 2078-2079.

Reglinski T, Rodenberg N, Taylor JT, Northcott GL, Ah Chee A, Spiers TM, Hill RA 2012. Trichoderma atroviride promotes growth and enhances systemic resistance in Diplodia pinea in radiata pine (Pinus radiata). Forest Pathology 42: 75-78. 